\title{
Fear shapes information acquisition in decisions from experience
}

\author{
Renato Frey ${ }^{\mathrm{a}, *}$, Ralph Hertwig ${ }^{\mathrm{a}}$, Jörg Rieskamp ${ }^{\mathrm{b}}$ \\ ${ }^{a}$ Max Planck Institute for Human Development, Berlin, Germany \\ ${ }^{\mathrm{b}}$ Department of Psychology, University of Basel, Switzerland
}

\section{A R T I C L E I N F O}

\section{Article history:}

Received 3 August 2012

Revised 5 March 2014

Accepted 26 March 2014

Available online 24 April 2014

\section{Keywords:}

Decisions from experience

Information search

Sampling

Emotional state

Fear

Risky choice

\begin{abstract}
A B S T R A C T
Before making decisions, people often need to explore their environment to learn about initially uncertain outcomes. To date, it remains unknown to what extent a person's emotional state shapes exploration in such decisions from experience. It has been suggested that fear regulates people's informational interface with the external world through its physiological expression (e.g., a more effective sampling of the visual field from widened eyes). We investigated whether-as suggested by appraisal tendency theories of emotions-the "emotional feeling" of fear triggers analogous changes in exploration, in terms of increased information sampling in decisions from experience. In two studies, one with naturally occurring emotional states and one with induced emotional states, we found that fearful (relative to happy) people sampled substantially more information before making a final choice. These different degrees of exploration influenced the experience of rarity and, in turn, final choices. We discuss the extent to which increased information acquisition is adaptive.
\end{abstract}

(c) 2014 Elsevier B.V. All rights reserved.

\section{Introduction}

When people make important decisions on matters such as their education, career, and relationships, they "must take account of many uncertain future possibilities" (Savage, 1954, p. 83). Only rarely do circumstances afford them the opportunity to consult descriptions of probability distributions over the myriad possible outcomes. These few exceptions include weather forecasts stating the likelihood of rain or other weather events (Gigerenzer, Hertwig, Van Den Broek, Fasolo, \& Katsikopoulos, 2005) and patient information leaflets listing a drug's potential side effects. Typically, however, people have to navigate the perils and opportunities of the environment without tabulated

\footnotetext{
* Corresponding author. Address: Max Planck Institute for Human Development, Center for Adaptive Rationality, Lentzeallee 94, 14195 Berlin, Germany. Tel.: +4930 82406472.

E-mail address: frey@mpib-berlin.mpg.de (R. Frey).
}

risks. They therefore need to draw on their past experience in similar situations (e.g., crossing busy streets)-if such experience exists-or they may engage in online exploration and search for information, thus ultimately making decisions from experience rather than decisions from description (Hertwig, Barron, Weber, \& Erev, 2004). In decisions from experience, the amount and the way people search for information can systematically influence their choices: For example, if a decision situation entails rare but potentially highly consequential events, people tend to learn about these events only by means of extensive and thorough exploration (Fox \& Hadar, 2006; Hau, Pleskac, Kiefer, \& Hertwig, 2008; Hertwig et al., 2004). But which factors shape exploration?

In order to map the psychology of search in decisions from experience, previous research has investigated the role of properties of individuals' cognition, such as working memory capacity (Rakow, Demes, \& Newell, 2008), numeracy, and rational thinking (Lejarraga, 2010). 
Additionally, researchers have examined the properties of the choice ecology (e.g., magnitude of incentives, loss versus gain domain, short- versus long-run frame; Hau et al., 2008; Lejarraga, Hertwig, \& Gonzalez, 2012; Wulff, Hills, \& Hertwig, submitted for publication) and the interaction of ecology and cognition (e.g., amplification effect; Hertwig \& Pleskac, 2010). While substantial progress has been made in describing how cognitive and ecological properties shape search and exploration in decisions from experience, there are still blind spots. One concerns the role of people's emotional states. Not only are emotions ever-present, they are likely to be intensified when people make decisions with uncertain outcomes. How do they shape exploration in decisions from experience? This article represents a first step towards answering this question.

\subsection{Darwin's view of emotions and information acquisition}

Emotional states appear to have considerable implications for an organism's information processing. Indeed, Darwin (2009/1872) already proposed that the facial expressions of specific emotional states interact with information processing. For instance, he suggested that the expression of surprise when startled has the following adaptive benefits:

As surprise is excited by something unexpected or unknown, we naturally desire, when startled, to perceive the cause as quickly as possible; and we consequently open our eyes fully, so that the field of vision may be increased, and the eyeballs moved easily in any direction (p. 1427).

Susskind et al. (2008) put this idea to the test and found that people's ability to sample sensory information from the environment was indeed increased when they staged expressions of fear (e.g., opening their eyes and raising their brows): Participants reported a subjectively larger visual field (relative to a neutral expression) and showed faster saccadic eye movements, which facilitated scanning of the environment and swift detection of potential threats.

Building on Susskind et al.'s (2008) notion that emotional facial expressions mediate the "sensory interface with the physical world" (p. 843), we examine the related possibility that "emotional feelings" (LeDoux, 1998)-the subjective counterpart of the physiological (e.g., expressive) component of emotions-may also contribute to regulating the decision maker's informational interface with the external world.

\subsection{Emotion as information and as informational regulator}

How emotions interact with cognitive processes has been extensively studied (for reviews, see Lench, Flores, \& Bench, 2011; Vohs, Baumeister, \& Loewenstein, 2007). One idea that has been particularly influential is that emotions, affects, and feelings themselves represent information providing input into cognitive processes like evaluations (including which information processing style to adopt; Schwarz \& Clore, 1983), choice, and inferences about uncertain environmental quantities such as risks and opportunities (e.g., Andrade \& Cohen, 2007; Bechara, Damasio, \& Damasio, 2000; Loewenstein, Weber, Hsee, \& Welch, 2001; Slovic, Flynn, \& Layman, 1991). Beyond being an important source of information, however, emotions may also regulate people's informational interface with the external world-this is the focus of the present article.

Emotions can occur incidentally as "background emotions" (i.e., unrelated to a current task; see Mellers et al., 2001, p. 267), be experienced online (e.g., the excessive fear that some people feel when they hear thunder; Arnauld \& Nicole, 1996; Loewenstein et al., 2001), or be anticipated to occur in the future (conditioned on the outcome of specific events; e.g., Mellers, Schwartz, Ho, \& Ritov, 1997). Here, we focus on background emotions. Although they are inescapable, little is known about how such emotional states shape information search (for exceptions, see Fiedler, Renn, \& Kareev, 2010; von Helversen \& Mata, 2012).

The baseline emotional state of most people is-fortunately-a mildly positive one. Across a surprising diversity of groups, "measurement methods in addition to selfreport indicate that most people's affect is primarily pleasant" (Diener \& Diener, 1996; Zelenski \& Larsen, 2000). Departures from this baseline state may prepare an organism for specific reactions to the environment-on a physiological and possibly also a cognitive level. That is, different emotional states involve specific states of action readiness (Frijda, 1986; Frijda, Kuipers, \& ter Schure, 1989). We focus on one specific deviation from this baseline emotional state, namely, fear. Specifically, as the physiognomy of fear facilitates visual search (Susskind et al., 2008), does a feeling of fear trigger analogous changes in search behavior, such as increased information acquisition via sampling from the world?

This possibility would conform with appraisal tendency theories of emotions (Lerner \& Keltner, 2000; Smith \& Ellsworth, 1985), according to which a person's emotional state leads to a specific cognitive appraisal of the environment and, in turn, to specific behavioral reactions. Cognitive appraisals are made along several dimensions, including perceived certainty (i.e., degree of subjective certainty about what is going on in the environment), situational control (i.e., degree to which a person feels the situation is controlled by circumstances versus by a human agent, including herself), and anticipated effort (i.e., degree to which a person feels that she needs to exert effort to deal with the situation). ${ }^{1}$ How does a fearful state-relative to a default (mildly positive) one-change a person's appraisals of the situation? Fear has been shown to trigger appraisals of low certainty, high situational control, and high anticipated effort (Smith \& Ellsworth, 1985). A plausible compensatory response to these appraisals would be more exploration of the environment. Let us consider how such a response would manifest in the context of decisions from experience.

\footnotetext{
${ }^{1}$ Different numbers of dimensions have been proposed. Roseman (1984) proposed five dimensions, Smith and Ellsworth (1985) eight. Of these, six were found to differ between emotions. For the present purpose, we focus on the three dimensions that pertain directly to search processes.
} 


\subsection{Exploration in decisions from experience}

Several experimental paradigms have been used to investigate decisions from experience (see Hertwig \& Erev, 2009). We rely on one of the most commonly used ones, the "sampling paradigm" (for details, see Hertwig et al., 2004). Specifically, decision makers are presented with two buttons on a computer screen, each representing an initially unknown payoff distribution. Before making a final incentivized choice, they can freely explore the payoff distributions by clicking the two buttons. Each click results in a random draw from the respective payoff distribution. Only after sampling as many outcomes as they like do participants decide on one of the payoff distributions and make a final consequential (i.e., incentivized) choice. The sampling paradigm represents a class of ecologies in which exploration and exploitation ${ }^{2}$ are separate (Hertwig \& Erev, 2009). Exploration unfolds during the sampling phase, and exploitation occurs at the moment of the final choice. There are two key markers of exploration in the sampling phase: sample size and switching frequency.

All other things being equal, larger samples will permit a more precise assessment of the properties of the underlying payoff distributions. Yet larger sample sizes also entail increased opportunity costs in terms of time and cognitive effort. One hypothesis linking fear and information acquisition is that fear-with its associated appraisal tendencies (low certainty, high situational control, and high anticipated effort)-prompts increased sampling: Large samples can help to attenuate uncertainty; they are also consistent with the high anticipated effort.

Exploration may also manifest in how a person organizes her search-in particular, in how frequently she switches between the payoff distributions during the sampling process. Hills and Hertwig (2010) distinguished between two paradigmatic search policies: In piecewise sampling, a person continuously switches back and forth between distributions (in the most extreme case, after every draw). In comprehensive sampling, a person samples extensively from one distribution before switching (in the most extreme case, only once) and sampling from the other. That is, exploration can occur either mostly between distributions or mostly within distributions.

Hills and Hertwig (2012) observed a strong negative correlation between sample size and switching frequency ${ }^{3}$ in the sampling paradigm $(r=-.38$ to -.54$)$, meaning that those who sampled more also switched less. If a fearful state prompts more sampling, it may thus also prompt less switching (i.e., more exploration within payoff distributions). However, it is unclear whether the link between sample size and switching frequency will persist in a fearful state. If exploration in the sampling paradigm manifests as switching between payoff distributions (see Gonzalez \& Dutt,

\footnotetext{
2 Exploration and exploitation are two separate processes that jointly characterize the inherent dynamics of search. Exploration means acquiring information in order to find out which action is more instrumental in obtaining future rewards or avoiding future losses. Exploitation means actually obtaining that reward.

${ }^{3}$ Switching frequency denotes the observed number of switches over the maximum number of possible switches (i.e., total sample size minus 1 ).
}

2012), a fearful state should prompt simultaneously more sampling and more switching (i.e., more exploration between payoff distributions).

In what follows, we investigate how fear shapes exploration in the sampling paradigm, with sample size and switching frequency serving as markers of exploration.

\section{Study 1: Naturally occurring fear and exploration "in the wild"}

The impact of fear on exploration can be studied either by taking advantage of naturally occurring emotional states or by inducing emotional states. Both approaches have advantages and disadvantages. The rationale of inducing emotional states in the laboratory is, of course, to have more experimental control. For instance, under the assumption that discrete emotional states can be induced independently of each other, any observed effects could be traced back exclusively to the emotional state induced. Yet, it is an open issue to what extent emotional states (in particular negative ones) can be induced independently of each other. Indeed, several induction techniques have been found to trigger covarying emotions (Izard \& Bartlett, 1972; Polivy, 1981; Westermann, Spies, Stahl, \& Hesse, 1996). We return to this issue in Study 2.

In Study 1, however, we trade the advantage of experimental control against that of the distinctiveness of naturally occurring emotional states. Experience sampling techniques have shown that in daily life, “... negative emotions appear to be experienced more distinctly than positive emotions" (Zelenski \& Larsen, 2000, p. 188). Although some naturally occurring negative emotional states tend to blend into each other (sadness, frustration, and anger), others-including fear-seem to emerge relatively independently. Capitalizing on this phenomenon, Study 1 investigates how natural emotional states influence exploration.

\subsection{Method}

To find people likely to be in a naturally fearful state, we approached patients in the waiting room of a hospital's oral surgery department $(n=23)$. To recruit a control group of people who were likely in a naturally happy state, we approached customers at the entrance to a stand-up comedy show $(n=26)$. The distributions of age, sex, and education in both groups were nearly identical (for demographics, see Table S1 in the supplementary material).

Participants received CHF 5 (USD 5.7) initial credit. In addition, they had the chance to win (or lose) payment contingent on their choices in four risky decision problems (see Table 1), which were presented in the sampling paradigm on tablet computers. Prior to each choice, participants could sample freely from both payoff distributions for as long as they liked. The order of problems and arrangement of payoff distributions (left versus right) were randomized. Participants then rated their emotional state on a short form of the PANAS-X (Röcke \& Grühn, 2003). Finally, they were informed about their total payoff. On average, they earned CHF 3.6 (range: CHF 0-33). 
Table 1

Decision problems (DP) used in Study 1, taken from Hertwig et al. (2004). $H=$ payoff distribution with the higher expected value (EV); $L=$ payoff distribution with the lower EV.

\begin{tabular}{|c|c|c|c|c|c|c|}
\hline \multirow[t]{2}{*}{ DP } & \multicolumn{2}{|c|}{ Payoff distributions } & \multicolumn{2}{|c|}{ Expected values } & \multicolumn{2}{|c|}{ Sample sizes } \\
\hline & $H$ & $L$ & $H$ & $L$ & Fearful & Happy \\
\hline 1 & $4, .8$ & $3,1.0$ & 3.2 & 3 & 45 & 8 \\
\hline 2 & $-3,1.0$ & $-32, .1$ & -3 & -3.2 & 37 & 12 \\
\hline 3 & $-3,1.0$ & $-4, .8$ & -3 & -3.2 & 47 & 11 \\
\hline 4 & $32, .1$ & $3,1.0$ & 3.2 & 3 & 39 & 8 \\
\hline
\end{tabular}

\subsection{Results}

We used a Bayesian approach to estimate the reported parameters: group means (i.e., the mode of the estimated posterior distribution), confidence intervals (i.e., the $95 \%$ highest posterior density interval, HDI), group differences (we refer to "credible" differences when the 95\% HDIs do not include 0 ), and effect sizes. This approach consists in (a) selecting an appropriate descriptive statistical model of the data (e.g., normal distribution), (b) postulating prior distributions of the parameters (we used vague priors that are quickly overruled by the data), and (c) updating those priors in the light of the data, using Bayes theorem (for the advantages of this approach and for further details, see Kruschke, 2011).

\subsubsection{Emotions}

Not surprisingly, the participants waiting for medical attention (henceforth "fearful" participants) reported feeling more fearful and less happy than did the participants at the stand-up comedy show (henceforth "happy" participants; see Fig. 1). The dominant self-reported emotional state of the latter group was "happiness." The fearful participants also reported feeling somewhat angrier and sadder than the happy participants-an incidence of the covariation of emotional states mentioned above (we return to this issue in Study 2). Nevertheless, the ratings

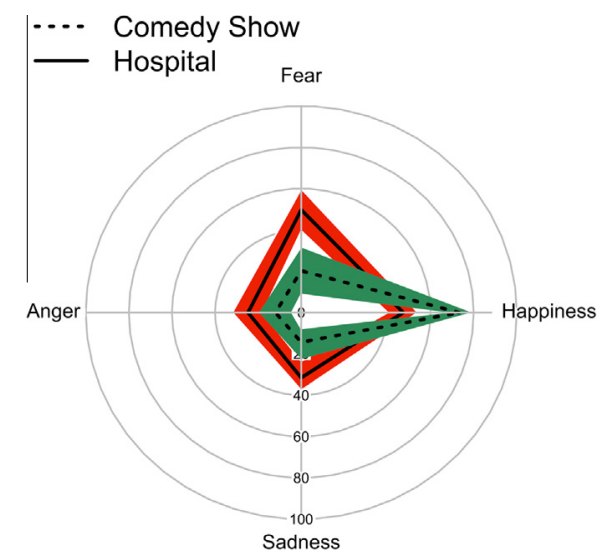

Fig. 1. Participants' self-ratings of their emotional states in Study 1 . The black dashed and solid lines depict the mean ratings (i.e., modes of the posterior distributions) and the shaded areas represent the 95\% HDIs. Participants in the hospital waiting room (solid line) were mostly fearful; participants at the comedy show (dashed line) were mostly happy. of the participants at the hospital clearly indicate that their emotional state was dominated by fear, with substantially higher ratings on this dimension than on anger or sadness.

\subsubsection{Search effort}

Averaged across all problems (Fig. 2A), fearful participants sampled substantially more than did happy participants, namely 45 times (95\% HDI $[27,59]$ ) versus 6 times (95\% HDI $[4,10])$. The effect size of the difference in sample size was very large $\left(\Delta M_{\text {draws }}=36,95 \%\right.$ HDI $[20,53]$, $d=1.6$ ). This finding supports the hypothesis that a fearful state, relative to a baseline happy state, leads to more extensive exploration and increased information acquisition in terms of larger sample sizes.

\subsubsection{Search policy}

Fig. 2B shows that fearful participants switched substantially less frequently between the two payoff distributions $(M=6 \%$, 95\% HDI [2\%, 14\%]) than did happy participants ( $M=96 \%, 95 \%$ HDI [92\%, 99\%]), reflecting a substantially different search policy $\left(\Delta M_{\text {switching }}\right.$ frequency $=90 \%, 95 \% \operatorname{HDI}[82 \%, 95 \%], d=13.4$ ).

We thus found that a fearful state, relative to a baseline happy state, prompts substantially larger sample sizes and more exploration within payoff distributions (i.e., less switching). That is, the two markers of exploration were negatively correlated $(r=-.82)$, as previously observed by Hills and Hertwig (2012).

Admittedly, a field study carries the risk of confounding variables. To rule out just one of them, we examined whether the patients awaiting medical attention had more time to kill than the customers awaiting the start of the comedy show, but found no such evidence (Supplementary material). To rule out other potential confounds, we next turned to experimentally induced emotional states.

\section{Study 2: Induced fear and exploration in the lab}

In this study, we adopted an emotion induction procedure, which-as noted above-is not without its own set of methodological challenges. Nevertheless, it promises the advantage of more experimental control. The main challenge of emotion induction procedures is to elicit discrete emotional states independently. Several emotion induction procedures (e.g., movies) have been found to trigger a pattern of covarying emotions (e.g., anger, disgust, and fear; Philippot, 1993; Polivy, 1981). Fear has proved particularly difficult to induce independently (in this case, with movie clips; Gross \& Levenson, 1995).

In Study 2, we tackled this challenge in the following way: As in Study 1, we sought to compare exploration in a group of fearful individuals relative to a control group of individuals in a baseline happy state. However, because the induction of fear may also activate other negative emotions, in particular anger and sadness (see Fig. 1), we included two additional conditions in which these two emotional states were explicitly induced. We were thus able to gauge the extent to which increased exploration in the fear condition of Study 1 was prompted by fear or co-determined by anger and sadness. 

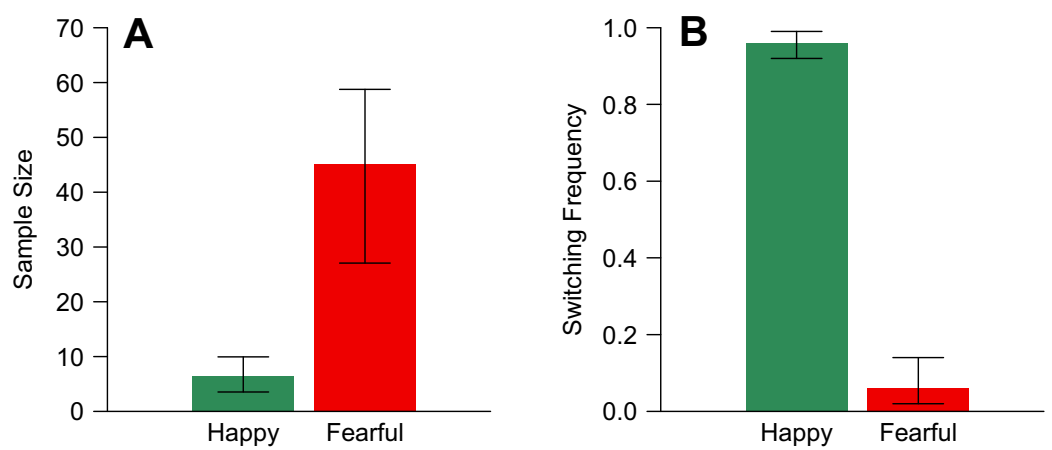

Fig. 2. Search effort and search policy in Study 1. Bars show the modes of the posterior distributions of sample size (panel A) and switching frequency (panel B) across the four decision problems (see Table 1). Error bars show 95\% HDIs.

\subsection{Method}

We recruited participants from the subject pool of the University of Basel's Department of Psychology (for demographics, see Table S2 in the Supplementary material) and randomly assigned them to one of four conditions: "happy" ( $n=27)$, "fearful" ( $n=29)$, "angry" ( $n=28)$, and "sad" $(n=28)$. Participants received a fixed fee of CHF 7.50 (or course credits) plus a performance-contingent payment, which ranged between CHF 0 and 20, and was on average CHF 4.9. At the outset, we measured participants' initial emotional states (using a finer grained version of the PANAS-X than in Study 1). Participants were then asked to remember and to write down life events in which they felt the target emotion. After two practice trials involving decisions from experience, we induced the desired emotional state as follows: Prior to the 1st, 4th, and 7th decision problem, participants were asked to vividly imagine the previously described life events for 1 min (see Brewer, Doughtie, \& Lubin, 1980; Schwarz \& Clore, 1983). We used the same four decision problems as in Study 1, supplemented by five new problems (Table 2). As before, participants could sample from the two payoff distributions for as long as they liked before making the final incentivized choice. Finally, we reassessed participants' emotional states and gave them outcome feedback.

\subsection{Results}

In all analyses, we used the Bayesian parameter estimation approach described in Study 1.

\subsubsection{Emotions}

We observed no differences in participants' initial emotional states across the four groups (Fig. 3, top row). As was to be expected, participants, on average, started the study in a fairly happy emotional state, consistent with the finding that most people's baseline emotional state is mildly positive (Diener \& Diener, 1996). In the manipulation check at the end of the study (Fig. 3, bottom row), participants in the control condition ("happy") reported a nearly identical pattern of emotional state as at the beginning. Participants in the "fearful" condition rated themselves as more fearful than at the beginning, but they also reported slightly increased levels of anger and sadness. Similarly, participants in the "angry" and "sad" conditions rated themselves as substantially angrier and sadder, respectively, than at the beginning of the study. In these two conditions, the emotion induction procedure also triggered covarying negative emotions to a certain degree-but only little fear. Contrasting these two additional conditions with the baseline happy state thus allows us to investigate the extent to which an angry or a sad emotional state leads to similar effects on exploration as does a fearful state.

Table 2

Decision problems (DP) used in Study 2. DPs 1-5 were taken from Hertwig et al. (2004), and DPs 6-9 were newly constructed. $H=$ payoff distribution with the higher expected value (EV); $L=$ payoff distribution with the lower EV.

\begin{tabular}{|c|c|c|c|c|c|c|c|c|c|c|}
\hline \multirow[t]{2}{*}{ DP } & \multicolumn{2}{|c|}{ Payoff distributions } & \multicolumn{2}{|c|}{ Expected values } & \multicolumn{2}{|c|}{ Sample sizes } & \multicolumn{2}{|c|}{ Rare event observations } & \multicolumn{2}{|c|}{ Higher EV choices } \\
\hline & $H$ & $L$ & $H$ & $L$ & Fearful & Happy & Fearful & Happy & Fearful & Happy \\
\hline 1 & $4, .8$ & $3,1.0$ & 3.2 & 3 & 42 & 37 & 4.66 & 4.33 & .59 & .52 \\
\hline 2 & $-3,1.0$ & $-32, .1$ & -3 & -3.2 & 56 & 40 & 3.03 & 2.26 & .38 & .15 \\
\hline 3 & $-3,1.0$ & $-4, .8$ & -3 & -3.2 & 58 & 35 & 6.1 & 4.56 & .69 & .3 \\
\hline 4 & $32, .1$ & $3,1.0$ & 3.2 & 3 & 57 & 34 & 3.03 & 1.78 & .41 & .26 \\
\hline 5 & $32, .025$ & $3, .25$ & .8 & .75 & 65 & 44 & 1.14 & 0.56 & .28 & .26 \\
\hline 6 & $3,1.0$ & $5, .55$ & 3 & 2.75 & 42 & 25 & 9.97 & 6.89 & .59 & .56 \\
\hline 7 & $11, .35$ & $4, .9$ & 3.85 & 3.6 & 49 & 30 & 3.03 & 1.07 & .52 & .41 \\
\hline 8 & $-12, .25$ & $-32, .1$ & -3 & -3.2 & 52 & 36 & 2.45 & 1.48 & .45 & .22 \\
\hline 9 & $-4, .25$ & $-3, .35$ & -1 & -1.05 & 55 & 31 & 6.55 & 4.07 & .48 & .56 \\
\hline
\end{tabular}



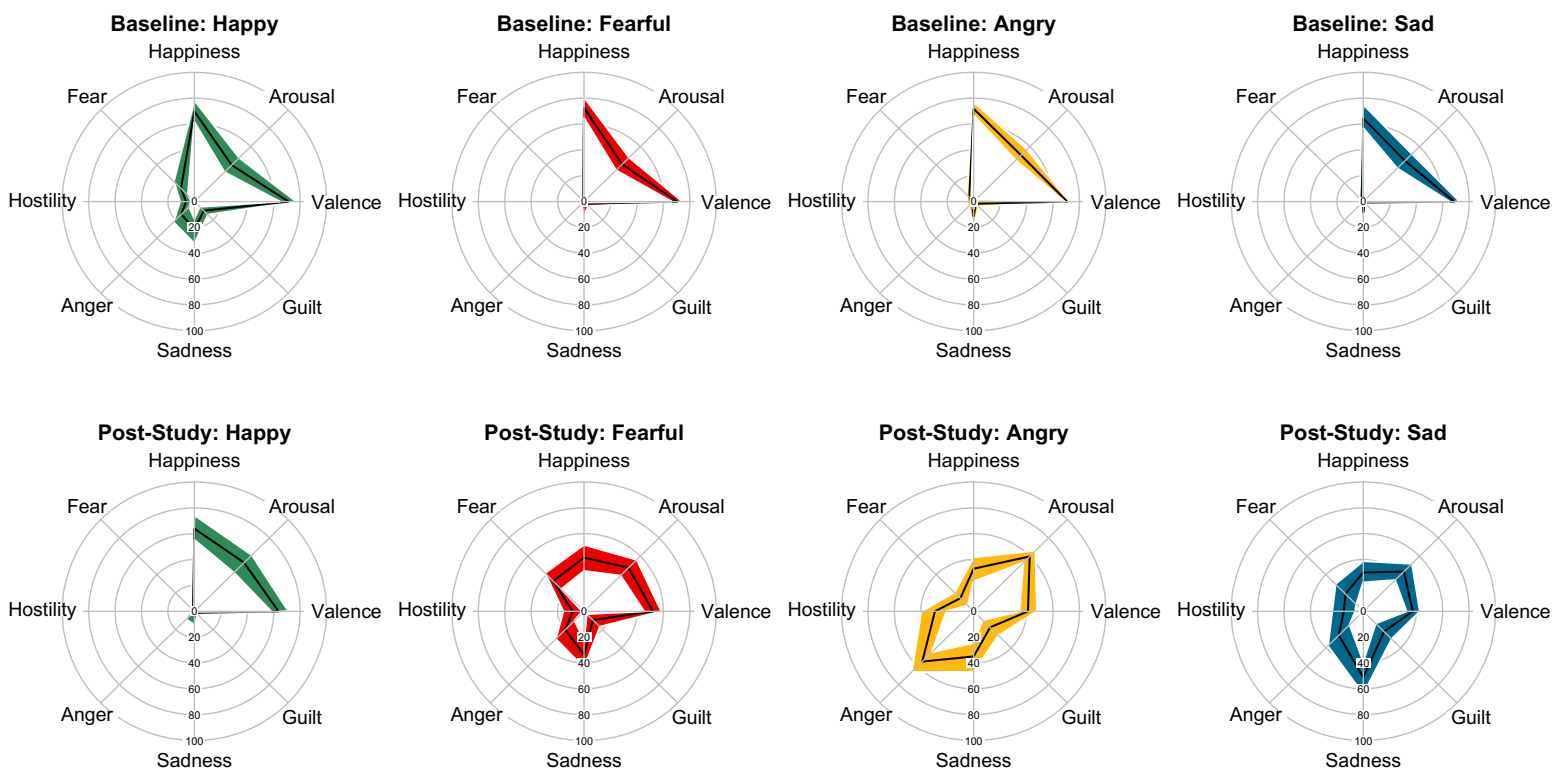

Fig. 3. Participants' self-ratings of their emotional states in Study 2. The top row shows the baseline ratings at the beginning of the study. The bottom row represents the manipulation check at the end of the study. Black lines depict the mean ratings (i.e., modes of the posterior distributions) and the shaded areas represent the $95 \%$ HDIs. In addition to the specific emotional states, we also asked participants to provide ratings for valence (positive and negative) and for arousal (high and low).

\subsubsection{Search effort}

Averaged across all problems (Fig. 4A), participants in the "fearful" condition sampled substantially more than did participants in the "happy" condition, namely 45 times (95\% HDI [36, 55]) versus 28 times (95\% HDI [20, 36])-an increase of $61 \%\left(\Delta M_{\text {draws }}=17,95 \%\right.$ HDI $\left.[5,30], d=.81\right)$. The other two conditions, "angry" and "sad," led to a slightly but not credibly increased search effort relative to the "happy" condition (angry: $\Delta M_{\text {draws }}=6,95 \%$ HDI $[-8,18]$, $d=.28$; sad: $\Delta M_{\text {draws }}=9,95 \%$ HDI $[-5,20], d=.38$ ). Relative to the "fearful" condition, search effort in the "angry" and "sad" conditions was not credibly different, but the effect sizes were medium (angry: $\Delta M_{\text {draws }}=-13,95 \%$ HDI $[-25,2], d=.53$; sad: $\Delta M_{\text {draws }}=-10,95 \%$ HDI $[-24$, $4], d=.47)$.

This result was corroborated in a mixed-effects linear regression model (random effects over participants) with sample size as dependent variable and condition and position of the decision problem (in the random sequence of the nine problems) as independent fixed effects. We included the position of the decision problem in the model to account for potential sequence effects (e.g., decreasing sample sizes across the experiment due to fatigue, boredom, or learning; see Lejarraga et al., 2012). Inclusion of this independent effect did not affect the other conclusions. We implemented the model using Markov Chain Monte Carlo (MCMC) methods with non-informative priors, using the MCMCglmm package for Bayesian generalized linear models (Hadfield, 2010). As Table 3 shows, the only credible predictor of sample size was the "fearful" condition. We did not find any evidence that sample size decreased across the experiment.

Beyond this systematic difference in search effort as a function of emotional states, we found that the sample
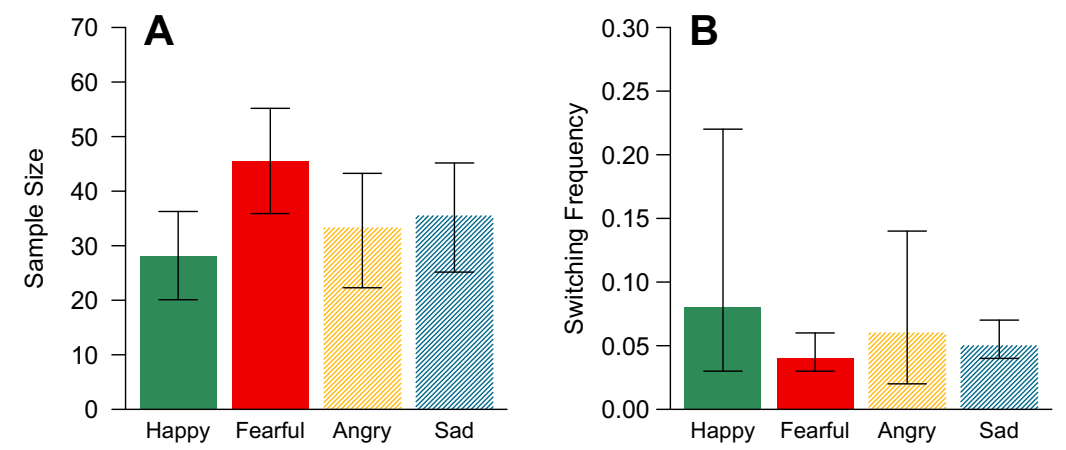

Fig. 4. Search effort and search policy in Study 2. Bars show the modes of the posterior distributions of sample size (panel A) and switching frequency (panel B) across the nine decision problems (see Table 2), separately for the different conditions. Error bars show 95\% HDIs. 


\section{Table 3}

Sample size and switching frequency in Study 2, predicted by the fixed effects "condition" and "problem position." The posterior beta weights were estimated using Markov Chain Monte Carlo sampling (MCMC); beta weights with HDIs that do not include 0 are printed in bold. HDI-l and HDI$\mathrm{u}$ stand for the lower and upper limits of the $95 \%$ highest density intervals, respectively.

\begin{tabular}{|c|c|c|c|c|c|c|}
\hline & \multicolumn{3}{|c|}{ Sample size } & \multicolumn{3}{|c|}{ Switching rate } \\
\hline & $b$ & HDI-l & HDI-u & $b$ & HDI-l & HDI-u \\
\hline \multicolumn{7}{|l|}{ Conditions } \\
\hline Intercept (happy) & 35.0 & 23.1 & 46.5 & .32 & .20 & .43 \\
\hline Fearful & 18.3 & 3.2 & 33.9 & -.13 & -.29 & -.02 \\
\hline Angry & 3.4 & -12.9 & 19.3 & .00 & -.16 & .15 \\
\hline Sad & 7.2 & -9.0 & 22.7 & -.11 & -.27 & .05 \\
\hline Problem position & -.1 & -.6 & .5 & .00 & -.01 & .00 \\
\hline
\end{tabular}

sizes in all conditions were substantially larger than those observed in Study 1 and in previous studies (see Hau, Pleskac, \& Hertwig, 2010, their Table 1). One key difference between this and previous implementations of the sampling paradigm is that presentation of the decision problems was interrupted several times by the emotion induction procedure. In a complementary analysis (presented in the Supplementary material), we pitted intermittent against continuous presentation of decision problems and observed that the interruptions in the sequence of decision problems are indeed likely to be responsible for the overall higher search effort. Given the overall large sample sizes, it is all the more noteworthy that a fearful state still resulted in substantially higher search effort than did the baseline happy state.

\subsubsection{Search policy}

Our analysis of search policy followed the same rationale as that of search effort. Averaged across all problems (Fig. 4B), participants in the "fearful" condition switched less between payoff distributions than did participants in the "happy" condition, namely, in 4\% (95\% HDI [3\%,6\%]) versus $8 \%(95 \% \mathrm{HDI}[3 \%, 22 \%])$ of choices. However, for participants in the "happy" condition, variance in switching frequency was high; consequently, the 95\% HDI for this difference just included $0\left(\Delta M_{\text {switching frequency }}=-4 \%, 95 \% \mathrm{HDI}\right.$ $[-18 \%, 1 \%], d=.53)$. Neither of the other two conditions differed credibly from the "happy" condition (angry: $\Delta M_{\text {switching frequency }}=-2 \%, 95 \%$ HDI $[-16 \%, 8 \%], d=.25$; sad: $\Delta M_{\text {switching frequency }}=-3 \%, 95 \%$ HDI $[-17 \%, 3 \%]$, $d=.39$ ).

In the mixed-effects linear regression model (Table 3), the "fearful" condition just failed to be a credible predictor of switching rate. The other conditions were clearly not credible predictors of switching rate.

To conclude, the results of Study 2 were mostly consistent with those of Study 1. A fearful state led to more exploration in terms of larger sample sizes. There was, albeit weak, evidence that fear also results in less switching, that is, more exploration within payoff distributions. As in Study 1, the link between sample size and switching frequency persisted. There was again a strong negative correlation between these two markers of exploration $(r=-.47)$.

\section{How more exploration affects choice}

In two studies, we observed that people in a fearful state explored more (in terms of larger sample sizes) than did people in a baseline happy state. Drawing large samples increases the likelihood of observing rare but potentially highly consequential events, which in turn may substantially influence choices. So did fearful and happy participants' choices differ? Before we turn to this question, let us first analyze participants' experience of rarity in the nine decision problems in Study 2.

\subsection{Experience of rarity}

As Fig. 5 shows, sample size and the experience of rare events was correlated in each decision problem and in both conditions. Furthermore, the increased exploration of participants in the "fearful" condition paid off: They observed the rare event in a decision problem on average 4.1 times (95\% HDI [3.1, 5.1]), whereas happy participants did so only 2.7 times (95\% HDI $[2.0,3.5])$-a difference of 1.4 times (95\% HDI [0.1, 2.6], $d=.62$ ). Participants in the "angry" and "sad" conditions experienced the rare events on average 2.9 (95\% HDI $[1.9,4.0])$ and 3 times (95\% HDI $[2.3,4.0])$, respectively. The differences to the "happy" condition of $0.2(95 \%$ HDI $[-1.0,1.5])$ and 0.3 times (95\% HDI $[-.8,1.4])$, respectively, were not credible.

\subsection{Choice}

All other things being equal, the choices of participants in the "fearful" condition can be expected to differ from those of the participants in the "happy" condition, to the extent that the former observed the rare events more often than the latter. However, it is possible that the fearful state not only prompted more exploration but also influenced participants' risk attitude. We investigated this possibility in a follow-up experiment (see Supplementary material) using the same emotion induction procedure and the same decision problems as in Study 2. To disentangle the issues of risk attitude and exploration, we presented the decision problems in a descriptive format. We were thus able to investigate the extent to which the emotional states of fear and the baseline happy state had diverging influences on risk preferences independent of exploration. The findings suggest that risk attitudes in these description-based choices did not differ as a function of the induced emotional states.

We therefore next tested the extent to which increased exploration and experience of rarity were associated with choosing the payoff distribution with the higher expected value (EV). As Fig. 6 shows, fearful participants indeed chose the payoff distribution with the higher EV credibly more often than happy participants did $\left(\Delta_{\text {higher EV }}\right.$ choices $=13 \%, 95 \%$ HDI $[3 \%, 23 \%], d=.65$ ). In contrast, participants in the "angry" and "sad" conditions, who also sampled slightly (although not credibly) more than participants in the "happy" condition, did not choose the payoff distribution with the higher EV credibly more often (angry: $\Delta_{\text {higher EV choices }}=7 \%, 95 \%$ HDI $[-2 \%, 16 \%], d=.44$; 

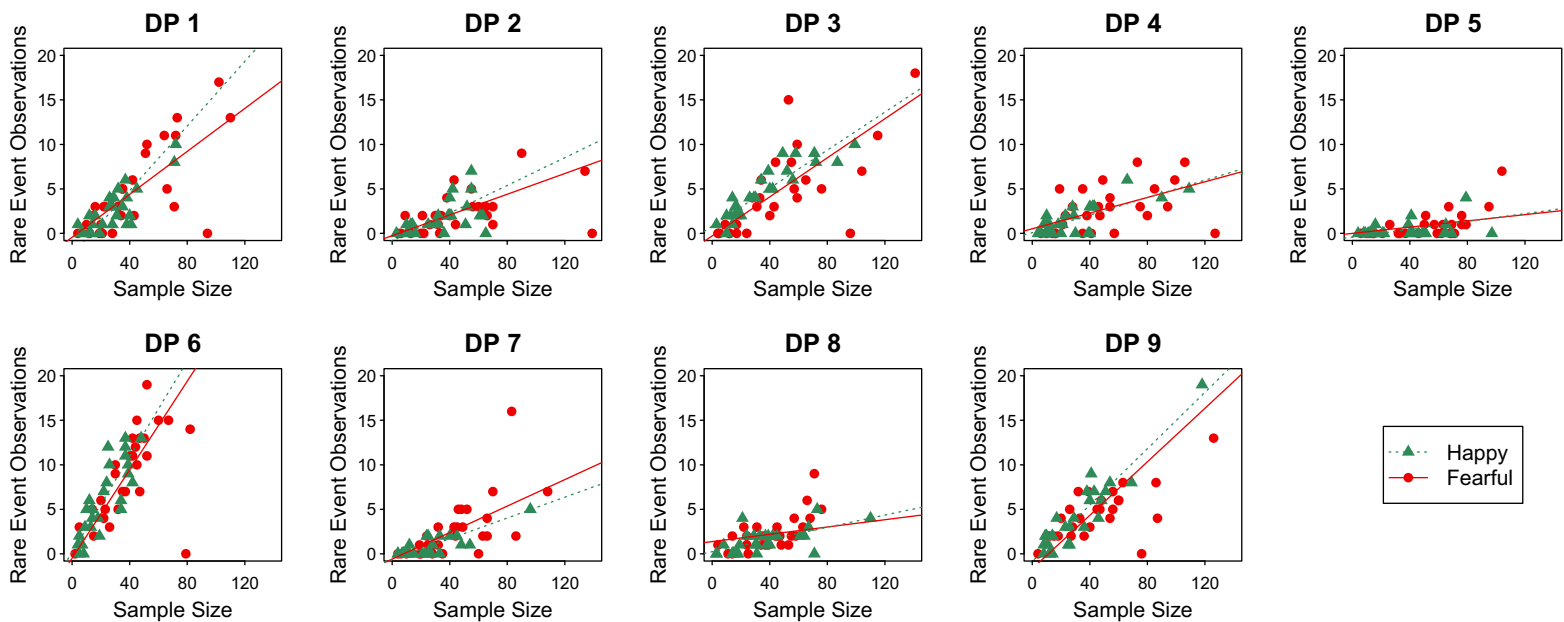

Fig. 5. Correlations between sample size and rare event observations in Study 2, separately for the nine decision problems (DP) and the participants in the "happy" versus "fearful" conditions. The data points for participants in the "angry" and "sad" conditions are omitted for simplicity, but the respective correlations are in line with the ones shown here.

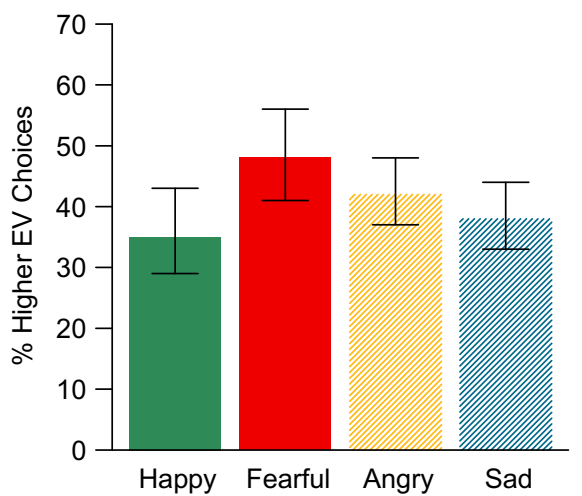

Fig. 6. Proportion of choices of the payoff distribution with the higher expected value (EV). Bars represent mean proportions per condition (i.e., modes of the posterior distributions), and error bars show 95\% HDIs.

sad: $\Delta_{\text {higher EV choices }}=2 \%, 95 \%$ HDI $\left.[-6 \%, 12 \%], d=.15\right)$ than did participants in the "happy" condition.

Taken together, these findings suggest that a fearful state, relative to a baseline happy state, prompts more exploration in terms of larger samples sizes, which in turn results in the more frequent experience of rare events and more choices in line with expected value maximization. This finding is similar to the observation by Wulff et al. (submitted for publication) that the cueing of a long-term frame (relative to a short-term frame) leads to more search and choices consistent with expected value maximization. Phenomenologically, a fearful state thus has the same effects as invoking a long-run frame.

\section{Discussion}

Investigating the influence of emotional states on cognition and decision making is not without problems. One problem concerns the extent to which people naturally experience discrete emotional states, and whether these can be induced independently of each other (see Izard \& Bartlett, 1972; Philippot, 1993; Polivy, 1981). Relatedly, people may not be perfectly accurate in reporting their current emotional state, and when prompted to do so (i.e., in manipulation checks), their ratings may reflect artificially blended emotional states (Polivy, 1981). We therefore chose two different approaches to investigate how emotional states shape exploration in the sampling paradigm, one employing naturally occurring emotions (Study 1 ) and one using induced emotions (Study 2). The results converged. First, in both studies, fearful participants sampled substantially more than did happy participants before making a final consequential choice. Second, during exploration, fearful participants tended to switch less often between payoff distributions than happy participants, suggesting more exploration within payoff distributions. Third, the difference in exploration led fearful participants to experience the rare events implicated in the decision problems more often, which in turn led them to choose the payoff distribution with the higher expected value more often, relative to happy participants.

In both studies, "fearful" participants also reported feeling somewhat angry and sad. In Study 2, we therefore also induced these two emotional states in separate conditions. The search and choice behavior observed in these two conditions was no different from that observed in the baseline happy state. Of the three negative emotions, it thus seems to be fear, in particular, that increases information acquisition (see also Susskind et al., 2008) and ultimately affects choice.

\subsection{How does happiness contribute to the pattern of results?}

The focus of this paper was on how a fearful state affects information acquisition. However, changes in the level of happiness may per se shape information acquisition in at least two ways. First, an increase in happiness 
(relative to the baseline happy state) could have the opposite effect of a fearful state, namely, decreased exploration. This possibility is difficult to address with our data: The emotion ratings of the "happy" participants at the comedy show in Study 1 matched the baseline ratings of the participants in Study 2. That is, participants at the entrance to the comedy show were likely not (yet) in a state of augmented happiness, relative to their baseline happy state. Further, the emotion induction procedure used in Study 1 maintained but did not increase "happy" participants' initial emotional states, which were already quite positive (see Fig. 3; this is a frequent observation-there is typically not much room for emotion induction procedures to boost happiness; Westermann et al., 1996). Therefore, it remains unclear whether augmented happiness would have reduced exploration relative to the baseline happy statethat is, would have had the opposite effect of fear. Nevertheless, the finding in Study 2 that explorative efforts did not differ across the "happy," "angry," and "sad" conditions offers no strong grounds to suspect that happiness lessened explorative impulses.

Second, attenuated happiness (relative to the baseline happy state) could theoretically lead people to explore less, thereby explaining the observed pattern of results. Specifically, the ratings for happiness in the "fearful" condition at the end of Study 2 were lower (Fig. 3) than those in the control condition (baseline happy state). In other words, happiness was "crowded out" by fear, and it is possible that this crowding out-rather than the increase in fearcaused more exploration. However, the crowding out of happiness also occurred in the "angry" and "sad" conditions (see Fig. 3), in which exploration was not increased relative to the control condition. Thus, we can rule out the possibility that increased exploration results from attenuated happiness.

\subsection{Limitations}

We investigated how specific departures from people's baseline happy state shaped their exploration. The tests we conducted thus used the "happy" condition as the point of reference and compared the three conditions representing specific negative emotional states against this baseline state. These comparisons revealed that fear had a credible effect on search and choice, but that anger and sadness did not. However, comparison of the "fearful," "angry," and "sad" conditions among each other showed that there were no credible differences in exploration and choice (see e.g., Section 3.2.2). What are the possible reasons and implications of this finding?

First, participants in the "angry" and "sad" conditions also rated themselves to be mildly fearful, an incidence of the covarying emotional states discussed above. These slight increases in fear may have caused a similar (but much weaker) effect as in the "fearful" condition. Second, the observed effect sizes (comparing the "angry" and "sad" conditions against the "happy" versus "fearful" conditions, respectively) suggest that the effects of anger and sadness were indeed more similar to the baseline happy state than to a fearful state. Third, the differences between both the "angry" and "sad" conditions and the "fearful" condition may have been larger were it not for the generally large sample sizes observed in Study 2 (which may have caused a ceiling effect, see Section 3.2.2). Clearly, more research is needed to shed light on the intricate relations among specific negative emotional states and their potential influence on exploratory behavior.

\subsection{Conclusions}

In sum, it appears that the facial expression of fear (see Susskind et al., 2008) and the subjective feeling of fear trigger parallel effects: acquisition of more information from the world. But is more information necessarily better? Cognitive psychologists and cognitive ecologists have recently begun to identify potential benefits of small samples and frugal search (see review in Hertwig \& Pleskac, 2010). In the context of decisions from experience, there is no simple answer as to how adaptive more search is. The reason is that the value of information depends on the statistical properties of the environment. One key property is rarity. The rarer the events representing a consequential loss or an attractive opportunity are, the more exploration increases the chance that an organism will encounter those events and thus become cognizant of their existence. All but two of the decision problems used in our studies involved rare events (i.e., events occurring with a probability smaller than .2; as defined in Hertwig et al., 2004). As shown in Study 2, participants in a fearful state not only explored more, they also observed the rare events more often, and made more choices favoring the payoff distributions with the higher expected value.

However, search in the typical monetary gambling environment is quickly subject to marginal decreasing utility of information (Hertwig \& Pleskac, 2010). Moreover, extensive information search in a non-stationary environment, relative to a stationary environment, confers even weaker adaptive benefits for the organism. The problem is, of course, that a person, at least initially, may not know what kind of environment he or she is navigating and may therefore decide-when feeling fearful-to err on the side of caution and to explore more.

\section{Acknowledgements}

We thank Susannah Goss and Laura Wiles for editing the manuscript, Eva Günther and Samuel Senn for collecting the data, and the Swiss National Science Foundation for a grant to the second author (100014-126558) as well as for a grant to the second and third author (CRSII1_136227).

\section{Appendix A. Supplementary material}

Supplementary data associated with this article can be found, in the online version, at http://dx.doi.org/10.1016/ j.cognition.2014.03.009.

\section{References}

Andrade, E. B., \& Cohen, J. B. (2007). Affect-based evaluation and regulation as mediators of behavior: The role of affect in risktaking, helping and eating patterns. In K. D. Vohs, R. F. Baumeister, \& 
G. Loewenstein (Eds.), Do emotions help or hurt decision making? A hedgefoxian perspective (pp. 35-68). New York, NY: Russell Sage Foundation.

Arnauld, A., \& Nicole, P. (1996). Logic or the art of thinking. Cambridge, UK: Cambridge University Press.

Bechara, A., Damasio, H., \& Damasio, A. R. (2000). Emotion, decision making and the orbitofrontal cortex. Cerebral Cortex, 10(3), 295-307. http://dx.doi.org/10.1093/cercor/10.3.295.

Brewer, D., Doughtie, E. B., \& Lubin, B. (1980). Induction of mood and mood shift. Journal of Clinical Psychology, 36, 215-226. http:// dx.doi.org/10.1002/1097-4679(198001)36:1<215::AIDJCLP2270360127>3.0.CO;2-6.

Darwin, C. (2009). The expression of the emotions in man and animals. New York, NY: Oxford (Original work published 1872).

Diener, E., \& Diener, C. (1996). Most people are happy. Psychological Science, $\quad 7(3), \quad$ 181-185. http://dx.doi.org/10.1111/j.14679280.1996.tb00354.x.

Fiedler, K., Renn, S. Y., \& Kareev, Y. (2010). Mood and judgments based on sequential sampling. Journal of Behavioral Decision Making, 23(5), 483-495. http://dx.doi.org/10.1002/bdm.669.

Fox, C. R., \& Hadar, L. (2006). "Decisions from experience" = sampling error + prospect theory: Reconsidering Hertwig, Barron, Weber \& Erev (2004). Judgment and Decision Making, 1, 159-161.

Frijda, N. H. (1986). The emotions. Cambridge, UK: Cambridge University Press.

Frijda, N. H., Kuipers, P., \& ter Schure, E. (1989). Relations among emotion, appraisal, and emotional action readiness. Journal of Personality and Social Psychology, 57(2), 212-228. http://dx.doi.org/10.1037/00223514.57.2.212.

Gigerenzer, G., Hertwig, R., Van Den Broek, E., Fasolo, B., \& Katsikopoulos, K. V. (2005). "A 30\% chance of rain tomorrow": How does the public understand probabilistic weather forecasts? Risk Analysis, 25(3), 623-629. http://dx.doi.org/10.1111/j.1539-6924.2005.00608.x.

Gonzalez, C., \& Dutt, V. (2012). Refuting data aggregation arguments and how the instance-based learning model stands criticism: A reply to Hills and Hertwig (2012). Psychological Review, 119(4), 893-898. http://dx.doi.org/10.1037/a0029445.

Gross, J. J., \& Levenson, R. W. (1995). Emotion elicitation using films. Cognition \& Emotion, 9(1), 87-108. http://dx.doi.org/10.1080/ 02699939508408966.

Hadfield, J. D. (2010). MCMC methods for multi-response generalized linear mixed models: The MCMCglmm R package. Journal of Statistical Software, 33, 1-22.

Hau, R., Pleskac, T. J., \& Hertwig, R. (2010). Decisions from experience and statistical probabilities: Why they trigger different choices than a priori probabilities. Journal of Behavioral Decision Making, 23, 48-68. http://dx.doi.org/10.1002/bdm.665.

Hau, R., Pleskac, T. J., Kiefer, J., \& Hertwig, R. (2008). The descriptionexperience gap in risky choice: The role of sample size and experienced probabilities. Journal of Behavioral Decision Making, 21, 493-518. http://dx.doi.org/10.1002/bdm.598.

Hertwig, R., Barron, G., Weber, E. U., \& Erev, I. (2004). Decisions from experience and the effect of rare events in risky choice. Psychological Science, 15, 534-539. http://dx.doi.org/10.1111/j.09567976.2004.00715.x.

Hertwig, R., \& Erev, I. (2009). The description-experience gap in risky choice. Trends in Cognitive Sciences, 13(12), 517-523. http:// dx.doi.org/10.1016/j.tics.2009.09.004.

Hertwig, R., \& Pleskac, T. J. (2010). Decisions from experience: Why small samples? Cognition, 115(2), 225-237. http://dx.doi.org/10.1016/ j.cognition.2009.12.009.

Hills, T. T., \& Hertwig, R. (2010). Information search in decisions from experience: Do our patterns of sampling foreshadow our decisions? Psychological Science, 21, 1787-1792. http://dx.doi.org/10.1177/ 0956797610387443.

Hills, T. T., \& Hertwig, R. (2012). Two distinct exploratory behaviors in decisions from experience: Comment on Gonzalez \& Dutt, 2011. Psychological Review, 119, 888-892. http://dx.doi.org/10.1037/ a0028004.

Izard, C. E., \& Bartlett, E. S. (1972). Patterns of emotions: A new analysis of anxiety and depression. Oxford, UK: Academic Press.

Kruschke, J. K. (2011). Bayesian assessment of null values via parameter estimation and model comparison. Perspectives on Psychological Science, 6(3), 299-312. http://dx.doi.org/10.1177/ 1745691611406925.

LeDoux, J. E. (1998). The emotional brain: The mysterious underpinnings of emotional life. New York, NY: Simon \& Schuster.
Lejarraga, T. (2010). When experience is better than description: Time delays and complexity. Journal of Behavioral Decision Making, 23(1), 100-116. http://dx.doi.org/10.1002/bdm.666.

Lejarraga, T., Hertwig, R., \& Gonzalez, C. (2012). How choice ecology influences search in decisions from experience. Cognition, 124, 334-342. http://dx.doi.org/10.1016/j.cognition.2012.06.002.

Lench, H. C., Flores, S. A., \& Bench, S. W. (2011). Discrete emotions predict changes in cognition, judgment, experience, behavior, and physiology: A meta-analysis of experimental emotion elicitations. Psychological Bulletin, 137(5), 834-855. http://dx.doi.org/10.1037/ a0024244.

Lerner, J. S., \& Keltner, D. (2000). Beyond valence: Toward a model of emotion-specific influences on judgement and choice. Cognition $\mathcal{E}$ Emotion, 14(4), 473-493. http://dx.doi.org/10.1080/ 026999300402763.

Loewenstein, G. F., Weber, E. U., Hsee, C. K., \& Welch, N. (2001). Risk as feelings. Psychological Bulletin, 127(2), 267-286. http://dx.doi.org/ 10.1037/0033-2909.127.2.267.

Mellers, B. A., Erev, I., Fessler, D. M. T., Hemelrijk, C. K., Hertwig, R., Laland, K. N., et al. (2001). Group report: Effects of emotions and social processes on bounded rationality. In G. Gigerenzer \& R. Selten (Eds.), Bounded rationality: The adaptive toolbox. Cambridge, MA: The MIT Press.

Mellers, B. A., Schwartz, A., Ho, K., \& Ritov, I. (1997). Decision affect theory. Psychological Science, 8(6), 423-429.

Philippot, P. (1993). Inducing and assessing differentiated emotionfeeling states in the laboratory. Cognition E Emotion, 7(2), 171-193. http://dx.doi.org/10.1080/02699939308409183.

Polivy, J. (1981). On the induction of emotion in the laboratory: Discrete moods or multiple affect states? Journal of Personality and Social Psychology, 41(4), 803-817. http://dx.doi.org/10.1037/00223514.41.4.803.

Rakow, T., Demes, K. A., \& Newell, B. R. (2008). Biased samples not mode of presentation: Re-examining the apparent underweighting of rare events in experience-based choice. Organizational Behavior and Human Decision Processes, 106, 168-179. http://dx.doi.org/10.1016/ j.obhdp.2008.02.001.

Röcke, C., \& Grühn, D. (2003). German translation of the PANAS-X. Unpublished manuscript, Free University of Berlin, Germany.

Roseman, I. J. (1984). Cognitive determinants of emotion: A structural theory. In P. Shaver (Ed.), Review of personality and social psychology. Emotions, relationships, and health (Vol. 5, pp. 11-36). Beverly Hills, CA: Sage.

Savage, L. J. (1954). The foundations of statistics (2nd ed.). New York, NY: Dover Publications.

Schwarz, N., \& Clore, G. L. (1983). Mood, misattribution, and judgments of well-being: Informative and directive functions of affective states. Journal of Personality and Social Psychology, 45(3), 513-523. http:// dx.doi.org/10.1037/0022-3514.45.3.513.

Slovic, P., Flynn, J. H., \& Layman, M. (1991). Perceived risk, trust, and the politics of nuclear waste. Science, 254(5038), 1603-1607. http:// dx.doi.org/10.1126/science.254.5038.1603.

Smith, C. A., \& Ellsworth, P. C. (1985). Patterns of cognitive appraisal in emotion. Journal of Personality and Social Psychology, 48(4), 813-838. http://dx.doi.org/10.1037/0022-3514.48.4.813.

Susskind, J. M., Lee, D. H., Cusi, A., Feiman, R., Grabski, W., \& Anderson, A K. (2008). Expressing fear enhances sensory acquisition. Nature Neuroscience, 11(7), 843-850. http://dx.doi.org/10.1038/nn.2138.

Vohs, K. D., Baumeister, R. F. \& Loewenstein, G. (2007). Do emotions help or hurt decision making? A hedgefoxian perspective. New York, NY: Russell Sage Foundation Publications.

Von Helversen, B., \& Mata, R. (2012). Losing a dime with a satisfied mind: Positive affect predicts less search in sequential decision making. Psychology and Aging, 27, 825-839. http://dx.doi.org/10.1037/ a0027845.

Westermann, R., Spies, K., Stahl, G., \& Hesse, F. W. (1996). Relative effectiveness and validity of mood induction procedures: A metaanalysis. European Journal of Social Psychology, 26(4), 557-580. http:// dx.doi.org/10.1002/(SICI)1099-0992(199607)26:4<557::AIDEJSP769>3.0.CO;2-4

Wulff, D. U., Hills, T. T., \& Hertwig, R. (2014). The impact of short- and long-run frames on search and choice in decisions from experience (submitted for publication)

Zelenski, J. M., \& Larsen, R. J. (2000). The distribution of basic emotions in everyday life: A state and trait perspective from experience sampling data. Journal of Research in Personality, 34(2), 178-197. http:// dx.doi.org/10.1006/jrpe.1999.2275. 